\title{
Brindar malas noticias en la sala de emergencias
}

Breaking bad news in the emergency department

\author{
María Consuelo Ascencio-Romero", Nicole Cervera-Montenegro", \\ Jerson André Vigo-Rabanal ${ }^{1}$, Franco Ernesto León-Jiménez ${ }^{2,3}$ \\ Alumnos de la escuela de medicina humana-Universidad Católica Santo Toribio de Mogrovejo. \\ ${ }^{2}$ Médico Internista- Epidemiólogo, Hospital Regional Lambayeque. \\ ${ }^{3}$ Docente, escuela de medicina humana-Universidad Católica Santo Toribio de Mogrovejo.
}

An Fac med. 2015;76(1):85-6 / doi:10.15381/anales.v76i1.11083

\section{Señor Editor:}

En el volumen 74 número 3, año 2013, de su Revista, el artículo de revisión titulado "Guía para comunicar a familiares la muerte repentina en emergencia" de Falconí ${ }^{(1)}$ llamó nuestra atención.

En medicina, en especial en medicina de emergencias y desastres, el médico frecuentemente se ve obligado a enfrentar situaciones difíciles; una de ellas es comunicar la muerte de un ser querido a un familiar. Las estrategias para enfrentar esta difícil situación no se enseñan en las facultades de medicina. Los cursos de ética y deontología impartidos en pregrado solo abordan esta temática tangencialmente. Una forma incorrecta de brindar la información y/o el no contar con un plan establecido pueden aumentar las situaciones de tensión y estrés, tanto en el profesional de la salud como en el familiar del paciente fallecido.

La formación en pregrado del médico se centra fundamentalmente en aspectos técnicos. El paradigma mecanicista de 'curar enfermedades' es el preponderante en aulas y pabellones de medicina. La realidad es que el profesional de la salud no trabaja con enfermedades sino con enfermos. Lamentablemente la formación en habilidades sociales en la mayoría de los médicos, en muchas partes del mundo, dista de ser la ideal ${ }^{(2)}$.
Un entrenamiento precoz durante el pregrado, en el contexto de escenarios clínicos, puede potenciar el desarrollo de estas habilidades comunicacionales. El uso de 'actores' o el entrenamiento entre los mismos estudiantes en situaciones de este tipo han demostrado la eficacia de estas estrategias ${ }^{(3)}$.

Dar malas noticias puede tener impacto tanto en el médico como en la persona que recibe la noticia ${ }^{(4)}$. Existe evidencia de que el nivel de estrés del médico, la calidad de su diagnóstico y la frecuencia de mala praxis están relacionadas con su capacidad para dar malas noticias ${ }^{(5)}$.

En el Perú, así como en todo el mundo, se ha recopilado las opiniones de los profesionales sobre cómo brindar noticias a través de protocolos. Pese a esto, el cómo actuar en emergencias al momento de dar malas noticias sigue siendo todavía un desafío ${ }^{(1)}$. El problema es que menos del 25\% de estas publicaciones están sustentadas en evidencia ${ }^{(2)}$. Tras realizar una búsqueda en Cochrane para determinar si existía alguna revisión sistemática que aborde este tema, solo se halló una revisión de $1998^{(6)}$, con heterogeneidad alta, siendo 6 de los 11 estudios considerados de calidad deficiente.

Al realizar una búsqueda en Medline mediante la estrategia de búsqueda how to break bad news, solo se encontró 6 ensayos clínicos. Al leer los resúmenes de estos estudios, solo dos de ellos eran ensayos clínicos hechos en residentes de medicina; en estos estudios se halló una diferencia a favor del grupo que recibió activamente un entrenamiento específico para brindar malas noticias en relación al grupo de residentes que solo recibió la formación habitual ${ }^{(7)}$.

Los familiares deberían ser informados respecto a la evolución de su paciente, así como de los procedimientos que se le realizan. Según la evidencia, lo que ellos más valoran en el médico a la hora de dar las malas noticias son: la actitud al brindar la noticia, la confidencialidad, la claridad del mensaje y la capacidad de responder a las preguntas de los familiares ${ }^{(8)}$. Algo importante a considerar es también el contar con un ambiente adecuado al momento de brindar la información del deceso.

Finalmente, en nuestra opinión, cada centro debería elaborar un protocolo propio para afrontar este problema, con el fin de evitar un negativo impacto psicológico en el familiar, facilitando la asimilación de la noticia. El contar con un protocolo establecido ayudaría a mejorar la relación médicofamiliar cuidador, reduciendo posibles malos entendidos por una información deficiente en la atención de los pacientes de emergencia. 


\section{REFERENCIAS BIBLIOGRÁFICAS}

1. Falconí E. Guía para comunicar a familiares la muerte repentina en emergencia. An Fac med. Perú [revista en la Internet]. 2013;74(3):217-9. Disponible en: http://www.scielo.org.pe/pdf/afm/ v74n3/a10v74n3.pdf

2. Vandekieft G. Breaking Bad News. American Family Physician. 2001;64(12):1975-3. Disponible en: http://www.ncbi.nlm.nih.gov/pubmed/11775763

3. Luttenberger K, Graessel E, Simon C, Sonath C. From board to bedside -training the communication competences of medical students with role plays. BMC Med Educ. 2014;14:135. doi: 10.1186/1472-6920-14-135.
4. Gao Z. Delivering bad news to patients-the necessary evil. J Med Coll PLA. 2011;26(2):103-8. DOI: 10.1016/S1000-1948(11)60033-6

5. Levinson W, Roter DL, Mullooly JP, Dull VT, Frankel RM. Physician-patient communication. The relationship with malpractice claims among primary care physicians and surgeons. JAMA. 1997 Feb 19;277(7):553-9.

6. Walsh RA, Girgis A, Sanson-Fisher RW. Breaking bad news 2: What evidence is available to guide clinicians?. Behav Med. 1998;24(2):61-72.

7. Head BA, Earnshaw LA, Greenberg RB. I will never forget: what we learn from medical student reflection of palliative care experience. J Palliat Med. 2012;15(5):535-41.
8. Jukovich GJ, Pierce B, Pananen L, Rivara FP. Giving bad news: the family perspective. J Trauma. 2000;48:865-73.

Carta recibida el 12 de enero de 2015.

Conflicto de interés: Los autores declaran no tener ningún conflicto de interés.

\section{Correspondencia:}

Jerson André Vigo Rabanal

Dirección: Av. Salaverry 650 Dpto. 405-B, Chiclayo,

Perú

Correo electrónico: jersonvigo@gmail.com 